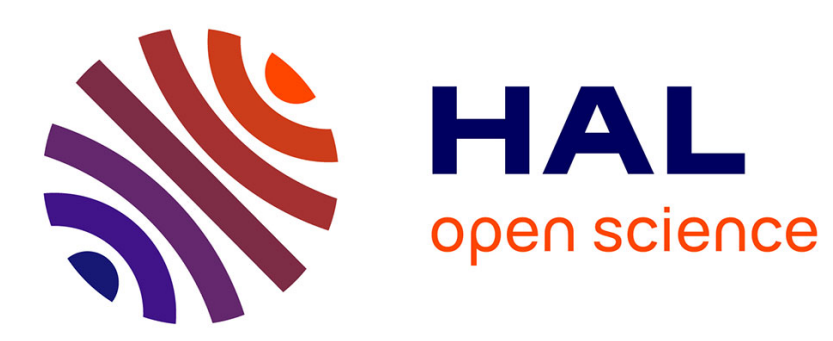

\title{
On the alignment dynamics of a passive scalar gradient in a two-dimensional flow
}

A. Garcia, M. Gonzalez, P. Paranthoën

\section{To cite this version:}

A. Garcia, M. Gonzalez, P. Paranthoën. On the alignment dynamics of a passive scalar gradient in a two-dimensional flow. Physics of Fluids, 2005, 17, pp.117102. 10.1063/1.2130750 . hal-01450279

\author{
HAL Id: hal-01450279 \\ https://hal.science/hal-01450279
}

Submitted on 31 Jan 2017

HAL is a multi-disciplinary open access archive for the deposit and dissemination of scientific research documents, whether they are published or not. The documents may come from teaching and research institutions in France or abroad, or from public or private research centers.
L'archive ouverte pluridisciplinaire HAL, est destinée au dépôt et à la diffusion de documents scientifiques de niveau recherche, publiés ou non, émanant des établissements d'enseignement et de recherche français ou étrangers, des laboratoires publics ou privés. 


\title{
On the alignment dynamics of a passive scalar gradient in a two-dimensional flow
}

\author{
A. Garcia, M. Gonzalez, and P. Paranthoën \\ CNRS UMR 6614/CORIA, Laboratoire de Thermodynamique, Site universitaire du Madrillet, \\ BP 12, 76801 Saint-Etienne du Rouvray Cedex, France
}

(Received 19 April 2005; accepted 14 September 2005; published online 10 November 2005)

\begin{abstract}
A Lagrangian study of the statistical properties of the orientation of a passive scalar gradient is performed using experimental data and a simple, numerical analysis. It is shown that, in a low-Reynolds number Bénard-von Kármán street, the temperature gradient downstream of a heated line source does not align with the asymptotic orientation predicted by the Lapeyre et al. model [Phys. Fluids 11, 3729 (1999)] in the hyperbolic regions. This result is ascribed to fluctuations of strain persistence along Lagrangian trajectories. A numerical analysis of the scalar gradient alignment properties shows that these fluctuations, together with a low level of the rate of strain, may lead to preferential orientations that are different from the theoretical one predicted by an asymptotic model. () 2005 American Institute of Physics. [DOI: 10.1063/1.2130750]
\end{abstract}

\section{INTRODUCTION}

The orientation of the gradient of a passive scalar with respect to the principal strain directions is an essential property of the scalar field, for it governs the evolution of the gradient magnitude. Alignment with the most compressive strain direction, by causing the rise of the scalar gradient, is the key mechanism of small scale production and cascade phenomena.

In three-dimensional (3D) turbulence, the statistics of a passive scalar gradient derived both from numerical simulations $^{1-3}$ and experiments ${ }^{4}$ reveal a preferential alignment with the compressional strain. Interestingly, recent studies of the two-dimensional (2D) case ${ }^{5,6}$ clearly show that in strain-dominated regions the scalar gradient actually does not tend to align with a strain eigenvector, but with a direction determined by the combined effects of strain and effective rotation (i.e., the sum of vorticity and the rotation rate of strain axes). Anyway, in both the 2D and 3D cases, it is only for the asymptotic, ideal pure strain that the compressional direction is a fixed point for the gradient orientation; the equilibrium alignment cannot coincide with any of the principal strain directions whenever rotation is present.

Now, even if an equilibrium orientation (whether it corresponds to a strain direction or not) can be defined, the alignment statistics depend on the dynamics of the scalar gradient. In a nonstationary velocity field, good alignment with an equilibrium direction can be expected only if the gradient time response is fast enough as compared to the velocity gradient fluctuations. The orientation dynamics of the scalar gradient, then, is an essential aspect of small-scale mixing in turbulent flows and has been addressed in some previous studies. ${ }^{3,6-8}$

The overall problem of scalar gradient alignment is certainly intricate. Because it somewhat simplifies the strain/ vorticity interaction, the $2 \mathrm{D}$ case makes it, to a certain extent, more tractable. In a 2D flow, moreover, one can make use of a topology-based model of the scalar gradient behavior, ${ }^{5}$ which, as far as we know, has no 3D counterpart. This is the approach we take to study nonstationary aspects of scalar gradient alignment. The response of the gradient to timedependent velocity derivatives, especially, is examined in two ways. First, experimental data obtained in a laminar 2D Bénard-von Kármán street are analyzed statistically and compared to the prediction of the Lapeyre et al. model. ${ }^{5}$ In this situation, the tracer is simply dispersed by a smooth $2 \mathrm{D}$ velocity field characterized by a single large scale. Different experimental studies have been devoted to the understanding of $2 \mathrm{D}$ transport and mixing. ${ }^{9,10}$ Here, the originality of the study is that from a geometrical approach, the experiment allows revisiting the alignment of the scalar gradient in a 2D flow with specific conditions. In such conditions it seems justifiable to ask to what extent the results verify the Lapeyre et al. theory and are comparable to results obtained in turbulent flow.

Motivated by this experiment, we study, then, the effect of strain persistence fluctuations on the scalar gradient alignment by means of simulations using a simple, numerical model. We propose a modified picture of the orientation of the scalar gradient in strain-dominated regions.

\section{SCALAR GRADIENT ORIENTATION IN THE LAPEYRE et al. MODEL}

In a 2D flow, defining the scalar gradient by $\mathbf{G}$ $=\rho(\cos \theta, \sin \theta)$ and assuming incompressibility, the equation for the gradient orientation is ${ }^{5,6}$

$$
\frac{d 2 \theta}{d t}=\omega-\sigma \cos \zeta+\mathcal{D}_{\theta},
$$

where $\omega$ is the vorticity and $\zeta=2(\theta+\phi)$. Angle $\phi$ defines the orientation of the strain principal axes and $\tan 2 \phi=\sigma_{n} / \sigma_{s} ; \sigma_{n}$ and $\sigma_{s}$ are, respectively, the normal and shear components of strain and $\sigma_{n}^{2}+\sigma_{s}^{2}=\sigma^{2}$. The term $\mathcal{D}_{\theta}$ represents the effect of diffusion upon the gradient orientation and is written as ${ }^{6,11}$ 


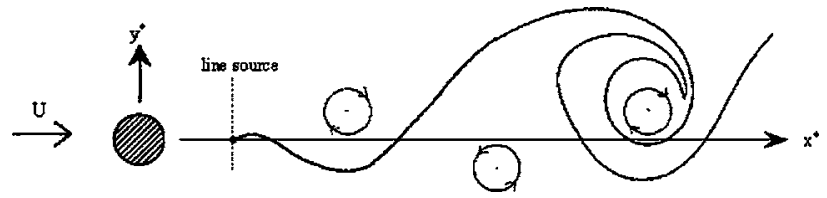

FIG. 1. Experimental setup.

$$
\mathcal{D}_{\theta}=2 D \Delta \theta+\frac{4 D}{\rho} \nabla \theta \cdot \nabla \rho,
$$

where $D$ is the molecular diffusivity.

Neglecting diffusivity, Eq. (1) is rewritten as ${ }^{5}$

$$
\frac{d \zeta}{d t}=\sigma(r-\cos \zeta)
$$

with $r$ given by

$$
r=\frac{\omega+2 d \phi / d t}{\sigma}
$$

This parameter defines strain persistence and had also been put forward by Dresselhaus and Tabor ${ }^{12}$ in the study of the alignment of material elements. Note that $r$ has a Lagrangian meaning and includes the acceleration gradient tensor through $d \phi / d t$, the rotation rate of the strain basis.

If slow variations of $r$ and $\sigma$ along Lagrangian trajectories are assumed, then the analysis of Eq. (3) shows that the evolution of the scalar gradient orientation depends on the flow topology in a rather simple manner. ${ }^{5}$ In straindominated regions $(|r|<1), \zeta$ tends to the stable fixed point, $\zeta_{-}=-\arccos (r)$, corresponding to an equilibrium orientation. For the special value $r=0$, the equilibrium orientation coincides with the compressional direction (i.e., $-\phi-\pi / 4)$ and $\zeta_{-}=\zeta_{c}=-\pi / 2$. In rotation-dominated regions $(|r|>1)$, there is no equilibrium orientation, but a most probable one that corresponds to the minimal rotation rate of the gradient and $\zeta_{\text {prob }}=[1-\operatorname{sign}(r)] \pi / 2$. Numerical simulations in 2D decaying turbulence ${ }^{5,6}$ have confirmed this behavior of the scalar gradient.

\section{TEMPERATURE GRADIENT BEHAVIOR IN A BÉNARD-VON KÁRMÁN FLOW}

\section{A. Experimental conditions}

The scalar gradient statistics have been experimentally studied in a simplified, 2D situation. As shown in Fig. 1, the flow considered here is the Bénard-von Kármán street, that is, the periodic wake of a circular cylinder at low Reynolds number Re. In this flow, a symmetric temperature field is generated using a small heated line source located in the near wake, on the centerline. ${ }^{13,14}$ Figure 2 presents the instantaneous velocity and temperature fields obtained at a given value of the phase and shows the smooth thermal plume development.

The cylinder and line source diameters are, respectively, $d=2 \mathrm{~mm}$ and $d_{s}=20 \mu \mathrm{m}$. The Reynolds number based on the cylinder diameter is $\operatorname{Re}=63$ (i.e., $\operatorname{Re}=\operatorname{Re}_{c}+15$, where $\operatorname{Re}_{c}$ is the critical Reynolds number) and the source Reynolds number is smaller than unity. The line source is heated so that

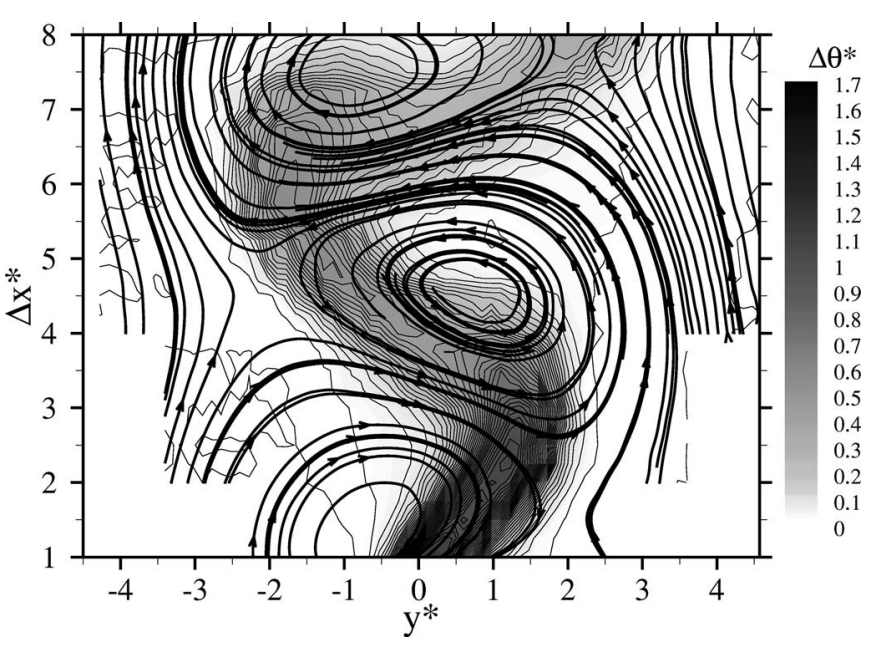

FIG. 2. Instantaneous velocity and temperature fields obtained at a given value of phase (streamlines in bold lines and temperature differences in gray scale).

buoyancy effects are avoided. In this experiment, the Richardson number, calculated with the Reynolds and Grashof numbers of the source, is lower than $10^{-3}$, which ensures that the thermal field is passive. ${ }^{14}$ The streamwise location of the line source is $x_{s}^{*}=x_{s} / d=7$. Caution is taken in controlling the initial conditions at the cylinder and to obtain a $2 \mathrm{D}$ velocity field. ${ }^{13}$ In the following, an asterisk denotes normalization. Lengths are normalized by the cylinder diameter, $d$, and velocities by the upstream velocity, $U_{\infty}$. Temperature differences are normalized by a reference excess temperature. ${ }^{14}$

Simultaneous measurements of the streamwise and transverse velocity components and of temperature have been achieved by using a two-component Laser Doppler Anemometry (LDA) system combined with a cold-wire probe. A phase reference for the LDA-cold-wire probe signals is taken from a second cold wire. Velocity and temperature gradients are derived from the measured velocity and temperature fields. More details on experimental conditions and measurement techniques are given in Refs. 13 and 14 .

\section{B. Statistics of the temperature gradient orientation}

The Lagrangian evolution of the temperature gradient has been derived by numerically tracking fictitious particles assumed to move at the measured velocity. The injection of particles is performed at 39 different points along the transverse section, located at $\Delta x^{*}=x^{*}-x_{s}^{*}=1$ downstream of the line source. Temperature and velocity at each point of a trajectory are interpolated from their respective experimental fields. Following this procedure, the temperature and velocity gradients along the trajectories also are derived and used to compute the quantities of interest such as vorticity, strain, $r$, temperature gradient modulus, and orientation (see the Appendix for details).

The data consist of 7800 trajectories of time duration $T_{0}=33 \mathrm{~ms}$ each (the Lagrangian time series are divided into 200 stages). The statistics of the temperature gradient alignment are derived from the Lagrangian sample. Weak gradients are discarded by conditioning for the dimensionless 


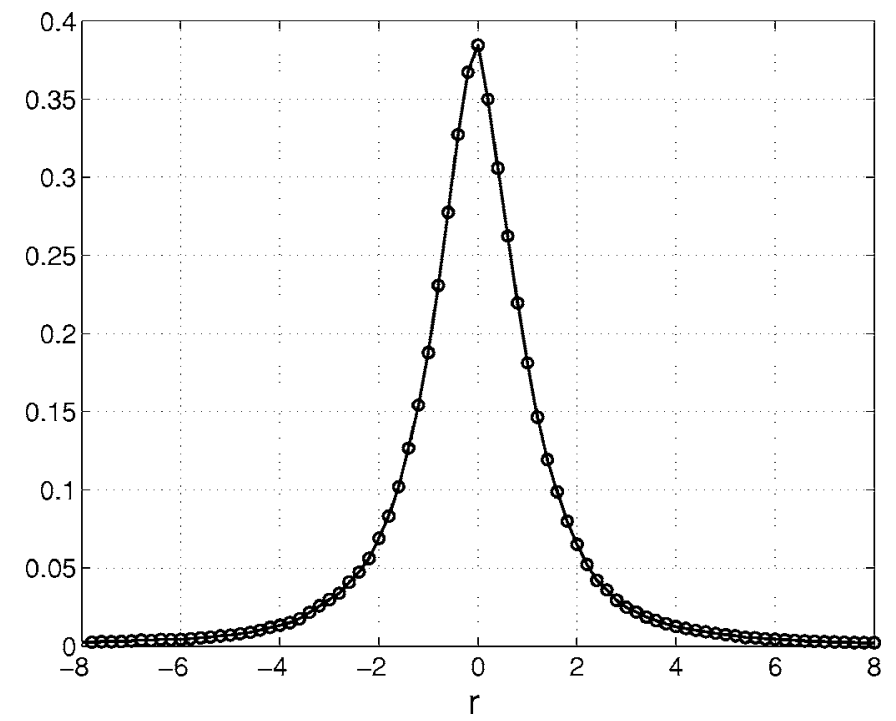

FIG. 3. pdf of $r$ where $|\mathbf{G}| \geqslant 100$.

norm $|\mathbf{G}|>100$. Sensitivity to this threshold is rather delicate to check because the scalar gradient tends to decay downstream and is correlated to the distance from the source. A too high threshold would imply considering the near field only. We consider that the chosen threshold is a good compromise between a reasonably large sample and a good representation of the passive scalar field in the experiment; we note that the largest gradient magnitude is about 3000. Incompressibility of the flow is used to improve the procedure and to ensure the validity of the velocity data. In the following, experimental statistics are performed imposing the criterion $\delta=|\partial u / \partial x+\partial v / \partial y| /(|\partial u / \partial x|+|\partial v / \partial y|)<0.2$.

Figure 3 presents the probability density function (pdf) of $r$ in the experiment and shows that the pdf is symmetric and centered on zero. Compared with numerical results in $2 \mathrm{D}$ turbulent flows, ${ }^{5}$ the pdf shape is quite different. Explaining this difference is not straightforward. It would require more details from both types of flow about quantities included in the parameter $r$, particularly concerning the statistics of the Lagrangian acceleration. We focus this study on hyperbolic regions and compare the alignments of the scalar gradient vector with the compressional strain axis and with the theoretical direction $\zeta_{-}$.

Close to the source, see Fig. 4(a), the probability density function of $\zeta-\zeta_{-}$has a maximum for zero. The pdf of $\zeta$ $-\zeta_{c}$, however, reveals a much better alignment of the temperature gradient with directions shifted by $\pi / 10$ from the compressional one. This is likely to result from the special condition of heat injection, which brings about preferential orientations of the thermal plume. The latter undergoes a flapping under the action of the local fluctuating transverse velocity, $v^{\prime}$. As already shown, ${ }^{14}$ the sinusoidal character of $v^{\prime} / U$ in the center of the vortex street implies that its pdf is symmetric with two peaks at $\pm\left(v^{\prime} / U\right)_{\max }$. Assuming that successive fluid particles coming from the source location keep their initial trajectories for times smaller than the Eulerian time scale of $v^{\prime} / U$, one can surmise that, in the near field of the source, the plume orientation pdf also is bimodal with
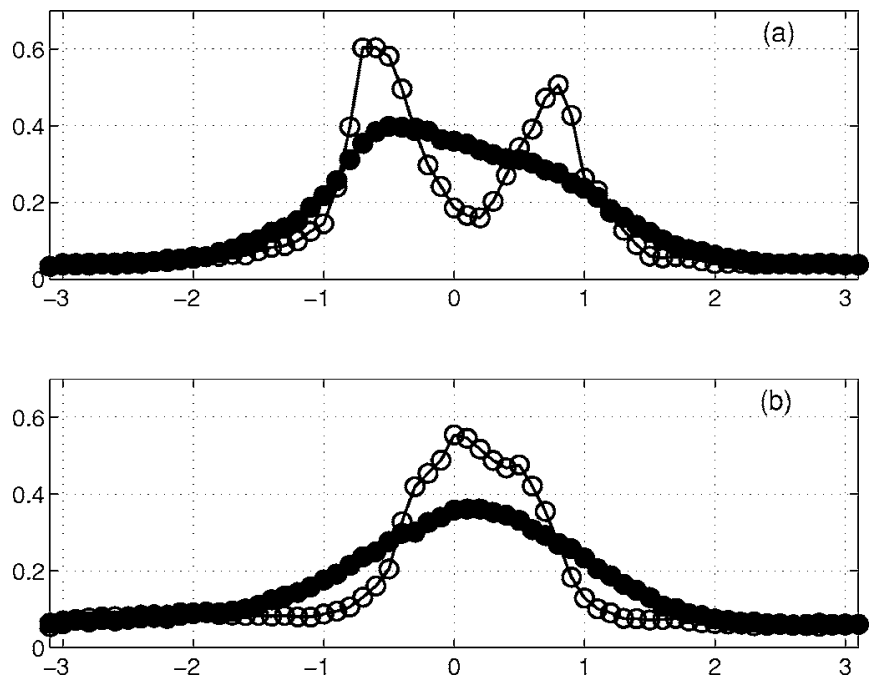

FIG. 4. Alignment pdf in the hyperbolic regions $(|r|<1)$ for $|\mathbf{G}| \geqslant 100$ : (a) "close" to the heat source $(\Delta x *<4)$; (b) "far" from the heat source $(\Delta x *>4) ; \bigcirc$ pdf of $\zeta-\zeta_{c} ; \bullet$ pdf of $\zeta-\zeta_{\text {. }}$.

peaks standing at $\pm \arctan \left(v^{\prime} / U\right)_{\max }$. It follows that the pdf of $2 \theta$ should have two peaks at $\alpha_{ \pm}= \pm 2 \arctan \left(v^{\prime} / U\right)_{\max }+\pi$, which has been checked (note that, because of the plume thickness, the angle $\theta$ defining the temperature gradient orientation in the experimental system of reference, is shifted by $\pm \pi / 2$ with respect to the local plume orientation). Now, the orientation of the temperature gradient with respect to the local strain axes also depends on angle $\phi$ (from the $\zeta$ definition). We have checked that angles $\alpha_{ \pm}$correspond to preferential orientations of $\phi$. The combination of these two orientations with respect to the compressional direction coincides with the shifted direction observed in the pdf.

Far from the source, see Fig. 4(b), the probability density function of $\zeta-\zeta_{c}$ shows that the temperature gradient loses the initial preferential orientation and tends to align with the compressional direction rather than with the asymptotic solution $\zeta_{-}$, which contrasts with the predictions of the Lapeyre et al. model.

\section{Analysis of experimental results}

The experimental case presented here shows that the orientation properties of the temperature gradient are different from those predicted by the Lapeyre et al. model in the hyperbolic regions. This could be ascribed to the memory of initial conditions, but this effect is unlikely. As displayed in Fig. 4, the alignment of the temperature gradient with the compressional direction is initially weak [Fig. 4(a)] and gets better farther downstream [Fig. 4(b)]. This behavior thus rather suggests the action of some mechanism promoting the compressional direction as the most probable one at the expense of the theoretical, asymptotic direction.

More insight into the right phenomenon is given by examining the Lapeyre et al. model. The latter is based on two main assumptions: (i) diffusion is neglected; (ii) $r$ and $\sigma$ vary slowly along Lagrangian trajectories (the analytical derivation of the gradient asymptotic orientation actually requires that $\sigma$ and $r$ are constant). The failure of at least one hypoth- 
TABLE I. Mean ratio of scalar gradient diffusion to mechanical effects $\left\langle D i_{\theta}\right\rangle$ conditioned on norm $\rho$ greater than $|\mathbf{G}|_{c}$.

\begin{tabular}{rcccc}
\hline \hline$|\mathbf{G}|_{c}$ & 100 & 200 & 500 & 800 \\
$\left\langle\boldsymbol{D} \boldsymbol{i}_{\theta}\right\rangle$ & 11.4 & 11 & 9.3 & 8.8 \\
\hline \hline
\end{tabular}

esis would explain the discrepancies between experimental data and model predictions and would reveal diffusion or nonstationary effects (or a combination of both mechanisms) in the experiment.

Analyses of diffusion influence upon scalar gradient alignment are scarce. It could be expected that diffusion generally causes misalignment as a result of blurring. In fact, nonlinear diffusion effects coupling the modulus and orientation of the gradient, see Eq. (2), make the real mechanism more intricate. As shown by Constantin et al. ${ }^{11}$ the nonlinear part of diffusion indirectly causes the destruction of weak, disorganized gradients while promoting alignment with the direction of the largest ones. Whether this mechanism is strong enough in comparison with linear diffusion effects, however, remains unclear. Anyway, the overall effect of diffusion upon the scalar gradient orientation should be Reynolds-number dependent. This is confirmed by numerical simulations in 2D turbulence. The numerical results of Lapeyre et al $^{5,6}$ for Reynolds numbers larger than $10^{4}$ show a weak effect of diffusion on gradient orientation. Insofar as the scalar gradient can be compared with the vorticity gradient, the simulations by Protas et al. ${ }^{15}$ in forced turbulence reveal that the vorticity gradient alignment with the compressional direction gets better as the Reynolds number is increased.

In the present low-Reynolds-number experiment, a nonnegligible influence of diffusion, then, is to be expected. To examine the importance of diffusion, we compute the mean ratio, $\left\langle D i_{\theta}\right\rangle$, measuring the competition between diffusion and mechanical effects, where

$$
D i_{\theta}=\sqrt{\frac{\mathcal{D}_{\theta}^{2}}{\omega^{2}+\sigma^{2}}} .
$$

The quantity $\left\langle D i_{\theta}\right\rangle$ is calculated from the experimental data for the scalar gradient norm $\rho$ larger than $|\mathbf{G}|_{c}$ (Table I).

We can see that diffusion is not negligible and that $\left\langle D i_{\theta}\right\rangle$ slightly decreases when $|\mathbf{G}|_{c}$ increases. These values are not surprising. An upper bound of $\left\langle D i_{\theta}\right\rangle$ can be estimated by computing the ratio of the convective time scale $\tau_{c}=d / 2 U_{\infty}$ to the scalar diffusion time scale, $\tau_{\theta}=d_{s}^{2} / D$, which leads to $\tau_{c} / \tau_{\theta} \simeq 100$.

This large level of diffusion, however, does not explain the difference between the experimental data and the Lapeyre et al. approach regarding the temperature gradient orientation. Figure 5 presents the $\zeta-\zeta_{c}$ and $\zeta-\zeta_{-}$pdf's far from the source, in the hyperbolic regions, with $\zeta$ computed from Eq. (3) in which $\sigma$ and $r$ are taken from the experimental data. Since Eq. (3) neglects diffusion, this is an indirect estimate of diffusion influence on gradient orientation. The pdf's derived following this procedure are sharper than the full-experimental ones [Fig. 4(b)], but still reveal a better

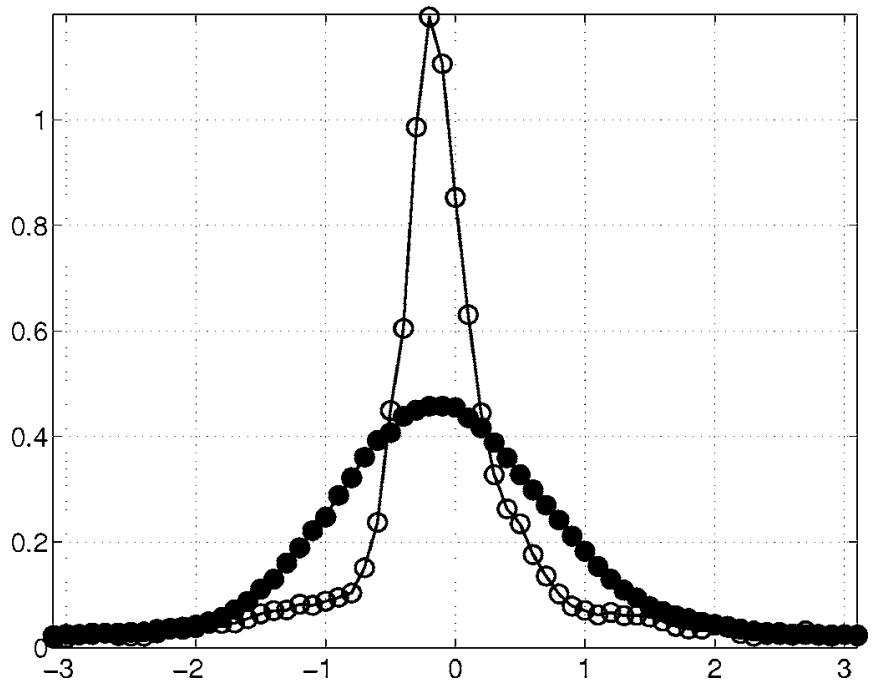

FIG. 5. Alignment pdf for $|r|<1$ and for $|\mathbf{G}| \geqslant 100$ derived from Eq. (3) in which $\sigma$ and $r$ are taken from the experimental data. $\bigcirc$ pdf of $\zeta-\zeta_{c}$; $\bullet$ pdf of $\zeta-\zeta_{-}$.

alignment with the compressional direction than with the Lapeyre et al. equilibrium orientation. A comparison of Figs. 4(b) and 5 shows that diffusion effects tend to move both alignments closer. It can be noted that the pdf's are slightly shifted toward negative values. This is due to the mean value of $r$, which is not exactly zero. Although, as revealed by $\left\langle D i_{\theta}\right\rangle$, diffusion is far from being negligible in this experiment, its action is not responsible for misalignment with the theoretical equilibrium direction. This lends support to the idea that nonstationary effects are likely to play the main role in misalignment.

We now examine the second assumption of the Lapeyre et al. model, stating that $\sigma$ and $r$ vary "slowly" along Lagrangian trajectories. An important issue consists in evaluating to what extent the possible nonvalidity of this assumption may change the picture of the alignment mechanism. A first clue is provided by the evolution of $\sigma, r$, and $d \phi / d t$ along a
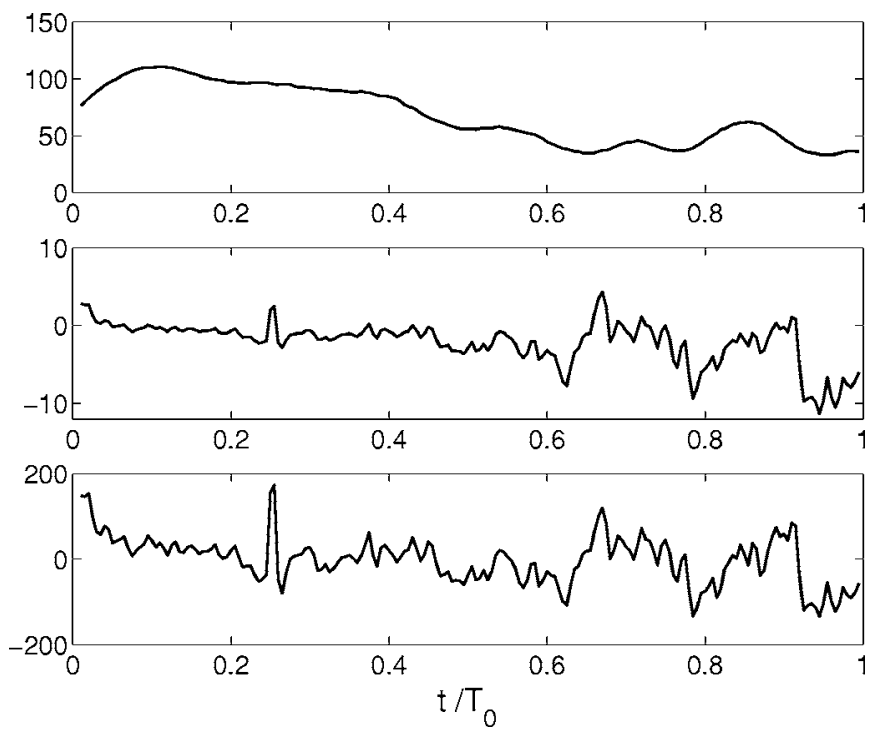

FIG. 6. Lagrangian evolution of $\sigma$ (top), $r$ (middle), and $d \phi / d t$ (bottom). 


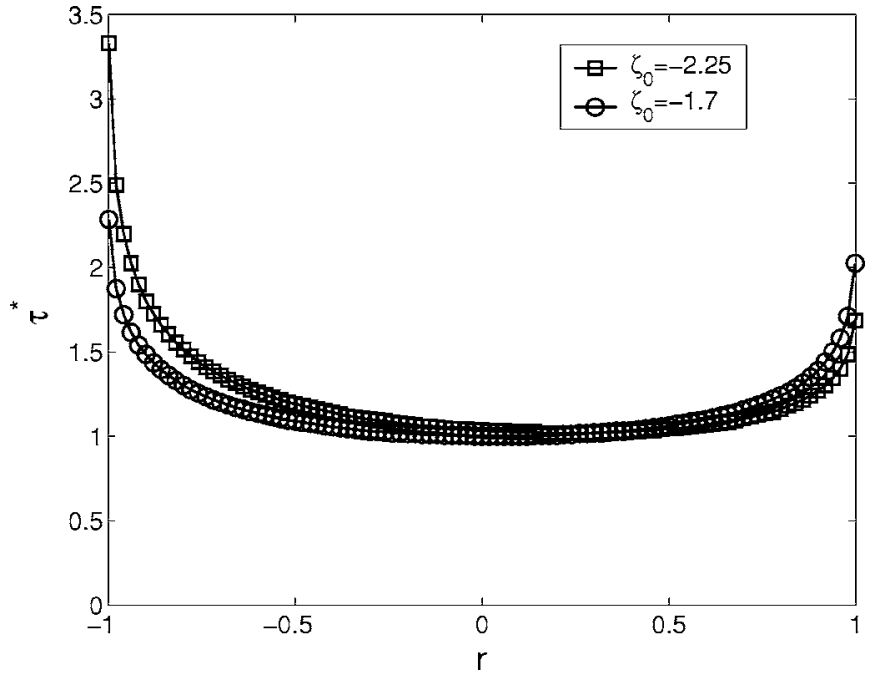

FIG. 7. Scalar gradient response time scale $\tau^{\star}$ as a function of $r$ for two initial orientations $\zeta_{0}$.

typical, experimental Lagrangian trajectory; see Fig. 6. It is clear that $r$ varies as $d \phi / d t$. We can notice, also, that $r$ varies on a shorter time scale than $\sigma$, which is understandable, for $d \phi / d t$ includes the Lagrangian derivative of $\sigma$. In the case under study, assuming the slow Lagrangian evolution of both $\sigma$ and $r$ may therefore be questionable.

Now, from the strict view of signal theory, Eq. (3) is nothing but the dynamic response of variable $\zeta$ to a timevarying forcing, $r$, with a relaxation time scale of the order of $1 / \sigma$. It thus follows that the key parameter is the ratio of the characteristic time scale of $r$ fluctuations (say, $T$ ) to the relaxation time scale; large $\sigma T$ imply that $\zeta$ responds to $r$ fluctuations and is always close to the equilibrium value (if any) imposed by the instantaneous value of $r$ whereas low $\sigma T$ bring about an inadequate response. This suggests that in the experiment, fluctuations of the strain persistence parameter, $r$, occurring on a time scale shorter than $1 / \sigma$ might lead to misalignment of the temperature gradient with respect to the theoretical orientation predicted by the Lapeyre et al. model.

The response time scale resulting from Eq. (3) is actually not $1 / \sigma$. Solving Eq. (3) for different values of $r$ shows that it is $r$ dependent, as depicted by Fig. 7 in which the response time scale normalized by $1 / \sigma, \tau^{\star}$, has been plotted for two different initial orientations of the scalar gradient. We have checked that the smallest value of $\tau^{\star}, \tau^{\star}=1$, which means that the smallest response time scale is $1 / \sigma$, occurs independently of the initial orientation. It follows, then, that the parameter $\sigma T$ overestimates the gradient response to $r$ fluctuations.

An order of magnitude of $\sigma T$ in the experiment is derived from the mean value of $\sigma,\langle\sigma\rangle \simeq 70 \mathrm{~s}^{-1}$ and the autocorrelation time scale of $r, T \simeq 2 \mathrm{~ms}$ (both quantities are obtained by averaging over all Lagrangian trajectories). The value $\sigma T \simeq 0.15$, which, as explained above, is overestimated, thus suggests that nonstationary effects should be significant in the experiment. In the following, the latter are examined by means of a simple numerical model. This model shows, incidentally, that it is not the time spent in
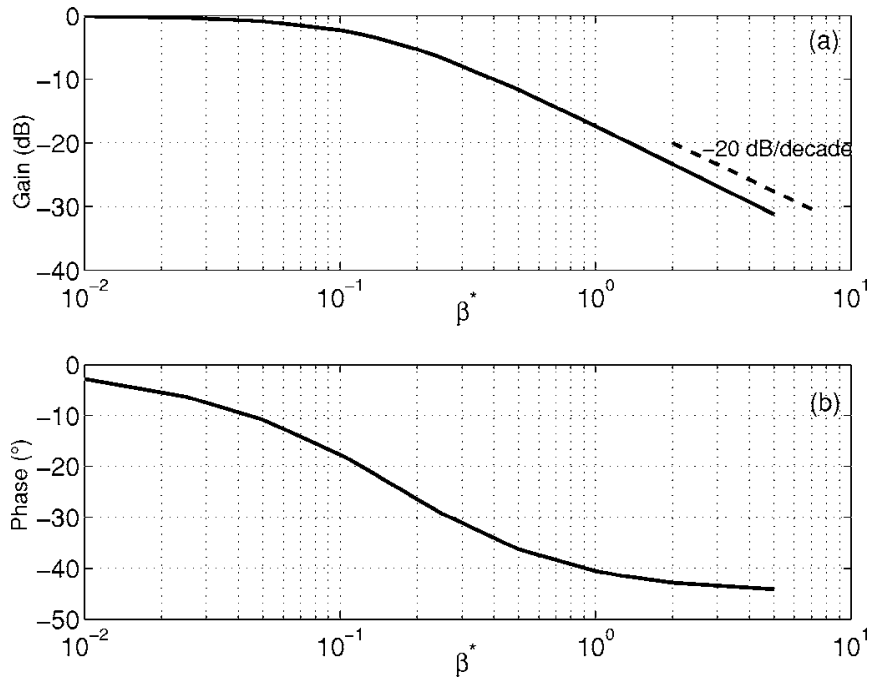

FIG. 8. Frequency response for gradient orientation: (a) gain $\mathcal{G}$; (b) phase $\psi$.

hyperbolic regions that explains the discrepancy between the experimental results and the prediction of the Lapeyre et al. model.

\section{ANALYSIS OF NONSTATIONARY EFFECTS UPON GRADIENT ALIGNMENT DYNAMICS USING A SIMPLE NUMERICAL MODEL}

In this section, the influence of the nonstationary effect on scalar gradient alignment is analyzed in the hyperbolic regime. Scalar gradient evolution has been numerically simulated using two different models for $r(t)$. Strain intensity, $\sigma$, is assumed to be constant and we introduce the dimensionless time $\tau=\sigma t$. The orientation equation [Eq. (3)] becomes

$$
\frac{d \zeta}{d \tau}=r-\cos \zeta .
$$

An additional orientation is defined as the orientation based on the mean value of $r$ instead of on its instantaneous value: $\zeta_{\langle r\rangle}=-\arccos \langle r\rangle$. Since the Lagrangian analysis of experimental data reveals that the probability density function of $r$ is symmetric and centered on zero (Fig. 3), the value chosen for the model is $\langle r\rangle=0$. In this case, the $\langle r\rangle$-defined orientation, $\zeta_{\langle r\rangle}$, coincides with the compressional orientation $\zeta_{c}$.

\section{A. The sinusoidal model for $\boldsymbol{r}$ time variations}

A sinusoidal model for $r$ is considered with the following expression:

$$
r(\tau)=\Delta r \sin \left(2 \pi \beta^{*} \tau\right),
$$

where the normalized frequency is $\beta^{*}=1 / \sigma T ; T$ is the oscillating period. Equation (4) describes a filter with input $r$ and output $\zeta$ and we examine how the scalar gradient orientation behaves when the input is sinusoidal.

Equation (4) is solved numerically with $\Delta r=0.8$ and the transfer functions are plotted in Fig. 8. Figures 8(a) and 8(b) display the frequency response in, respectively, gain and phase. The form of the transfer function, see Fig. 8(a), indi- 


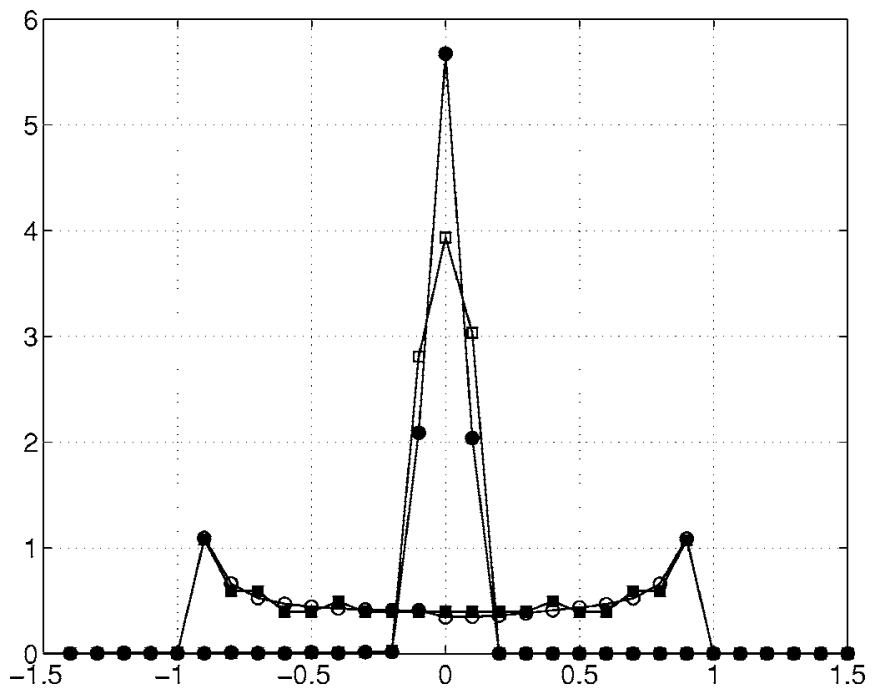

FIG. 9. Alignment pdf for $|r|<1$ with $\Delta r=0.8$ : with $\beta^{*}=2$; $\bullet$ pdf of $\zeta-\zeta_{\langle r\rangle} ; \mathbf{\square d f}$ of $\zeta-\zeta_{-} ;$with $\beta^{*}=0.01 ; \bigcirc$ pdf of $\zeta-\zeta_{\langle r\rangle} ; \square$ pdf of $\zeta-\zeta_{-}$.

cates that the scalar gradient orientation dynamics is represented by a first-order lag relation, but this is not true if phase is considered; see Fig. 8(b). The scalar gradient orientation behaves approximately as a low-pass frequency filter. This means that when $\beta^{*}$ is small, the scalar gradient orientation evolves as $\zeta_{-}$(determined by the instantaneous value of $r$ ), whereas for high values of $\beta^{*}$, the scalar gradient cannot keep up with $r$ time fluctuations, and its orientation is governed by $\langle r\rangle$.

It is also interesting to focus on the preferential alignment of the scalar gradient derived from this model. The probability density functions of $\zeta_{-} \zeta_{-}$and $\zeta_{-} \zeta_{\langle r\rangle}$ are calculated for two different normalized frequencies $\beta^{*}=0.01$ and $\beta^{*}=2$. It is worth noticing, from Fig. 9, that alignment properties directly depend on the value of $\beta^{*}$. For $\beta^{*}=0.01$, the $\zeta-\zeta_{-}$pdf reaches a maximum for zero, which means that the scalar gradient tends to align with the asymptotic direction. For $\beta^{*}=2$, the situation is opposite; the scalar gradient tends to better align with the $\langle r\rangle$-defined orientation $\zeta_{\langle r\rangle}$ than with the asymptotic direction.

We can summarize the main features of the scalar gradient orientation with parameter $\Gamma=P_{-}(X \in[-\varepsilon,+\varepsilon]) / P_{\langle r\rangle}(X$ $\in[-\varepsilon,+\varepsilon]$ ), where $P_{\langle r\rangle}$ and $P_{-}$are the probabilities of $\zeta$ $-\zeta_{\langle r\rangle}$ and $\zeta_{-} \zeta_{-}$and, arbitrarily, $\varepsilon=\pi / 10$. Figure 10 displays the evolution of $\Gamma$ vs $\beta^{*}$ for two different values of $\Delta r$. The scalar gradient moves from preferential alignment with the asymptotic solution $(\Gamma>1)$ to alignment with the compressional direction $(\Gamma<1)$ for $\beta^{*}$ values in the range $[0.1 ; 0.2]$.

This study was performed with $\Delta r<1$, but similar results concerning the orientation properties in the hyperbolic regime are obtained with $\Delta r$ greater than one. This model confirms that the parameter $\beta^{*}$ plays a crucial role in the alignment properties. High values of $\beta^{*}$ involve significant nonstationary effects, which lead to a better alignment with another direction than $\zeta_{-}$. As suggested by the numerical results, the preferential orientation, then, is $\zeta_{\langle r\rangle}$, which, for $\langle r\rangle=0$, coincides with the compressional direction.

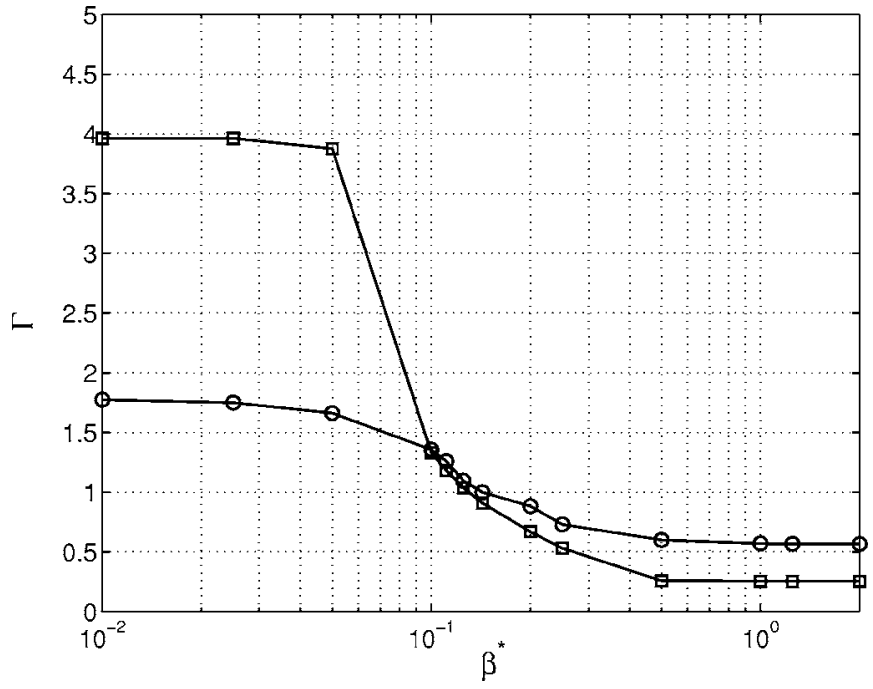

FIG. 10. Ratio $\Gamma$ measuring alignment with either $\zeta_{-}$or $\zeta_{\langle r\rangle}: \square, \Delta r=0.4$; O, $\Delta r=0.8$.

This numerical study, which may model any Lagrangian evolution of orientation, shows that the scalar gradient does not align with the equilibrium direction if nonstationary effects, betokened by $r$ fluctuations, are large; it rather tends to align with a direction determined by $\langle r\rangle$ and thus by the statistics of the flow topology.

\section{B. The stochastic model for $r$ time variations}

If a nonstationary behavior akin to turbulent fluctuations is to be simulated, then a more elaborated model than the previous one is required. Since $r$ includes velocity derivatives and the acceleration gradient tensor, its statistics is certainly not Gaussian. ${ }^{17}$ This is confirmed by the pdf of $r$ (Fig. 3 ). For the only purpose of examining the gradient orientation response to time-varying $r$, we, however, use a stochastic model based on a one-dimensional generalized Langevin equation. ${ }^{16}$ Parameter $r$, then, obeys a stochastic differential equation with zero mean and variance $r^{\prime 2}$ :

$$
d r(\tau)=-r \beta^{*} d \tau+\left(2 r^{\prime 2} \beta^{*}\right)^{1 / 2} d W(\tau) .
$$

Again, $\beta^{*}=1 / \sigma T$ where, this time, $T$ is the Lagrangian integral time scale and $d W(\tau)$ is a Wiener process.

As previously, the dimensionless equation, Eq. (4), is solved numerically using the $r$ signal derived from Eq. (5) and the statistics of $\zeta-\zeta_{\langle r\rangle}$ and $\zeta_{-} \zeta_{-}$are computed. According to Fig. 11, the scalar gradient tends to align preferentially with the $\langle r\rangle$-based direction rather than with the asymptotic solution $\zeta_{-}$. Figure 12 shows that for small values of $\beta^{*}$ $\left(\beta^{*}<0.5\right)$, the gradient is aligned with the direction corresponding to the asymptotic solution $(\Gamma>1)$ whereas for larger values, it reveals a much better alignment of the scalar gradient with the $\langle r\rangle$-based direction $(\Gamma<1)$.

Comparisons of computed results obtained from both previous models enable us to assess the significant role of nonstationary effects upon gradient scalar dynamics. Results concerning scalar gradient orientation in the experiment are consistent with these numerical results. Moreover, the results 


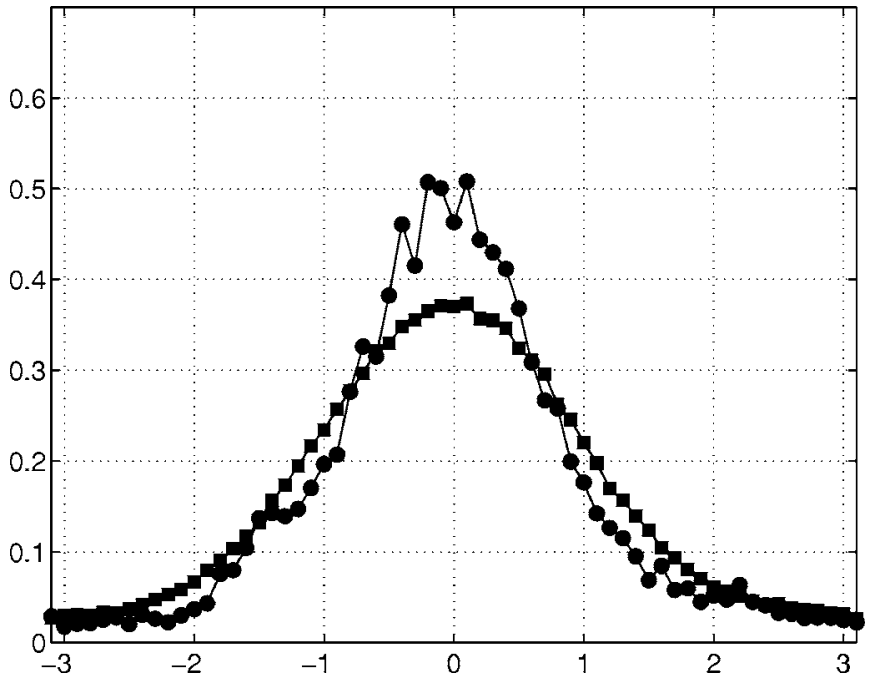

FIG. 11. Alignment pdf for $|r|<1$ with $\beta^{*}=5$ and $r^{\prime 2}=4$; $\bullet$ pdf of $\zeta-\zeta_{\langle r\rangle}$; udf of $\zeta-\zeta_{-}$.

described in this section show that when nonstationary effects through $r$ fluctuations are significant, the scalar gradient alignment strongly depends on $\langle r\rangle$ and, hence, on $r$ statistics. According to these results based only on the pdf of $r$ with $\langle r\rangle=0$, it would be interesting to explore further the influence of different $\langle r\rangle$ on the preferential alignment for imposed high value $\beta^{*}$.

Also, the numerical study reveals that the scalar gradient does not align with the theoretical orientation whatever the duration of the simulation, even for $r$ fluctuating with the constraint $|r|<1$, provided $\beta^{*}$ is large. This implies that when $r$ fluctuations are fast enough, the theoretical orientation is never reached, even if the time spent in hyperbolic regions is long.

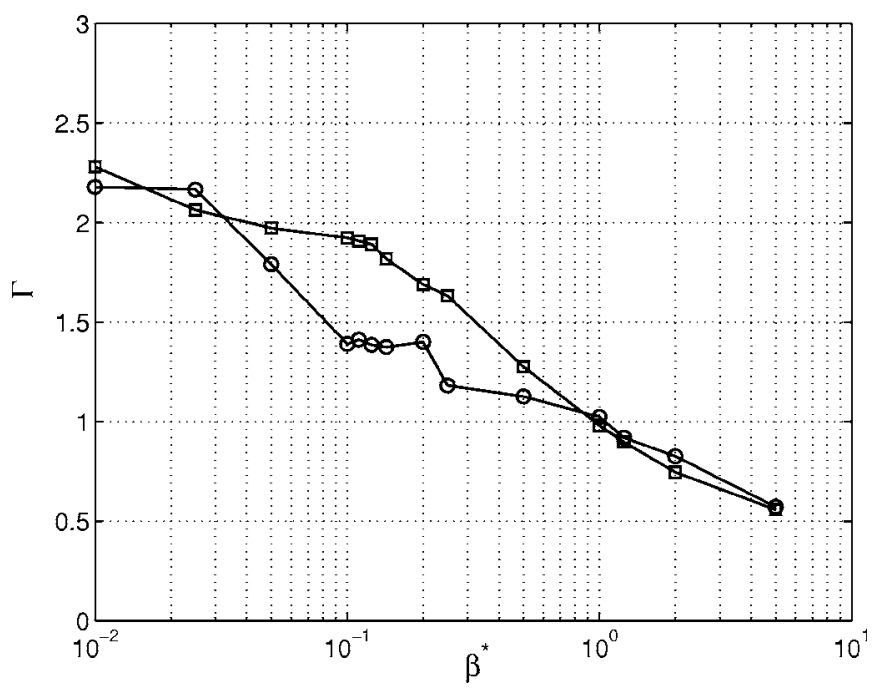

FIG. 12. Ratio $\Gamma$ measuring alignment with either $\zeta_{-}$or $\zeta_{\langle r\rangle}: \square, r^{\prime 2}=0.25$; $\mathrm{O}, r^{\prime 2}=4$.

\section{CONCLUSIONS AND DISCUSSION}

The Lagrangian evolution of the orientation of the temperature gradient downstream of a heated line source located in the center of a Bénard-von Kármán street has been studied using experimental data. The analysis has been focused on hyperbolic regions. In the latter, the experimental results are at variance with the Lapeyre et al. approach, which predicts that in strain-dominated regions the scalar gradient aligns with an asymptotic orientation $\zeta_{-}$, determined by $r$ and, in general, different from the compressional direction. Although diffusion has been checked to be significant in the orientation equation, it has been proved, however, that it is not diffusion that implies misalignment with $\zeta_{-}$. It has thus been inferred that, in this experiment, the alignment of the temperature gradient is noticeably influenced by nonstationarity through Lagrangian fluctuations of strain persistence. This explains that the Lapeyre et al. model, based on the assumption of slow Lagrangian variations of $r$, is not suited to this case.

The nonstationary effect upon scalar gradient alignment has also been examined using a simple numerical model, allowing for Lagrangian variations of $r$. The latter predicts that the scalar gradient aligns with the asymptotic orientation $\zeta_{-}$if $\sigma T \gg 1$, where $\sigma$ is the strain rate and $T$ is a characteristic time scale of $r$ fluctuations. If $\sigma T$ is weak, the scalar gradient tends to align with a direction that is determined by $\langle r\rangle$ and coincides with the compressional direction for $\langle r\rangle$ $=0$. These results add new considerations on the Lapeyre et al. model. They reveal that the scalar gradient alignment strongly depends both on $r$ statistics and the value of criterion $\sigma T$. The present study actually shows that the Lapeyre et al. model is valid, even for fluctuating $r$, provided $\sigma T$ is large enough.

A consequence of these results is that a moderate level of strain does not necessarily implies poor mixing provided $\sigma T$ is small and $\langle r\rangle$ is close to zero, for these conditions drive the scalar gradient to align with the compressional direction. It would be interesting to know if such conditions can be met in other types of flow.

To what extent the picture derived from the study of this low-Reynolds number flow can be extrapolated to turbulent flows and why the predictions of the Lapeyre et al. model are verified in a simulated turbulent flow (whereas they are not in the present one) are, also, important questions. Good predictions of the Lapeyre et al. model in turbulent flows might be explained by large levels of $\sigma T$, but checking this would require estimating the level of $\sigma T$ in $2 \mathrm{D}$ turbulent flow. As far as we know, there is no information concerning the Lagrangian characteristic time scale, $T$, of $r$ fluctuations. It would be interesting to derive this quantity from numerical simulations of turbulence, e.g., through the Lagrangian autocorrelation time scale of $r$. Moreover, this question is actually even more intricate because $d \phi / d t$ includes $\sigma$, which might imply that $T$ and $\sigma$ are, to some extent, not completely independent. For these reasons, further information would be appreciated to check our interpretation in turbulent flow.

\section{ACKNOWLEDGMENT}

The authors would like to thank Dr. G. Godard for his advice in using his experimental database. 


\section{APPENDIX: PROCEDURE FOR THE LAGRANGIAN CALCULATION OF DYNAMICAL QUANTITIES}

- From the Eulerian velocity and temperature dataset, the full set of first-order velocity and temperature space derivatives is calculated using a second-order scheme.

- Lagrangian trajectories are integrated with a second-order Runge-Kutta scheme using a particle velocity interpolated from the Eulerian velocity field by bicubic spline interpolation.

- Bicubic spline interpolation is also used to compute all other quantities (scalar gradient components, $\sigma_{n}, \sigma_{s}, \omega, \ldots$ ) along Lagrangian trajectories. In particular, the interest of this Lagrangian approach is mainly to calculate the $r$ parameter, for it includes the time derivative of the local frame orientation, $d \phi / d t$. The latter term is calculated along particle trajectories using the following relation:

$2 \frac{d \phi}{d t}=\frac{\sigma_{s}\left(d \sigma_{n} / d t\right)-\sigma_{n}\left(d \sigma_{s} / d t\right)}{\sigma^{2}}$

(derived from $\tan 2 \phi=\sigma_{n} / \sigma_{s}$ ) in which the derivatives of $\sigma_{n}$ and $\sigma_{s}$ are computed at the second order.

${ }^{1}$ W. T. Ashurst, A. R. Kerstein, R. M. Kerr, and C. H. Gibson, "Alignment of vorticity and scalar gradient with strain rate in simulated Navier-Stokes turbulence," Phys. Fluids 30, 2343 (1987).

${ }^{2}$ P. Vedula, P. K. Yeung, and R. O. Fox, "Dynamics of scalar dissipation in isotropic turbulence: a numerical and modeling study," J. Fluid Mech. 433, 29 (2001).

${ }^{3}$ G. Brethouwer, J. C. R. Hunt, and F. T. M. Nieuwstadt, "Micro-structure and Lagrangian statistics of the scalar field with a mean gradient in isotropic turbulence," J. Fluid Mech. 474, 193 (2003).

${ }^{4}$ B. Galanti, G. Gulitsky, M. Kholmyansky, A. Tsinober, and S. Yorish,
"Joint statistical properties of fine structure of velocity and passive scalar in high Reynolds number flow," in Advances in Turbulence X, Proceedings of the 10th European Turbulence Conference, edited by H. I. Andersson and P.-Å. Krogstad, Trondheim, Norway, 2004.

${ }^{5}$ G. Lapeyre, P. Klein, and B. L. Hua, "Does the tracer gradient vector align with the strain eigenvectors in 2D turbulence?" Phys. Fluids 11, 3729 (1999).

${ }^{6}$ G. Lapeyre, B. L. Hua, and P. Klein, "Dynamics of the orientation of active and passive scalars in two-dimensional turbulence," Phys. Fluids 13, 251 (2001).

${ }^{7}$ P. Klein, B. L. Hua, and G. Lapeyre, "Alignment of tracer gradient vectors in 2D turbulence," Physica D 146, 246 (2000).

${ }^{8}$ W. D. Smyth, "Dissipation-range geometry and scalar spectra in sheared stratified turbulence," J. Fluid Mech. 401, 209 (1999).

${ }^{9}$ E. M. Ziemniak, C. Jung, and T. Tel, "Tracer dynamics in open hydrodynamical flows as chaotic scattering," Physica D 76, 123 (1994).

${ }^{10}$ M.-C. Jullien, P. Castiglione, and P. Tabeling, "Experimental observation of Batchelor dispersion of passive tracers," Phys. Rev. Lett. 85, 3636 (2000).

${ }^{11}$ P. Constantin, I. Procaccia, and D. Segel, "Creation and dynamics of vortex tubes in three-dimensional turbulence," Phys. Rev. E 51, 3207 (1995).

${ }^{12}$ E. Dresselhaus and M. Tabor, "The kinematics of stretching and alignment of material elements in general flow field," J. Fluid Mech. 236, 415 (1991).

${ }^{13}$ G. Godard, "Etude expérimentale de la structure du champ d' un scalaire passif dans une allée de Bénard-von Kármán," Ph.D. thesis, University of Rouen, 2001.

${ }^{14}$ P. Paranthoën, G. Godard, F. Weiss, and M. Gonzalez, "Counter gradient diffusion vs. counter diffusion temperature profile," Int. J. Heat Mass Transfer 47, 819 (2004)

${ }^{15}$ B. Protas, A. Babiano, and N. K.-R. Kevlahan, "On geometrical alignment properties of two-dimensional forced turbulence," Physica D 128, 169 (1999).

${ }^{16}$ S. B. Pope, "Lagrangian pdf methods for turbulent flows," Annu. Rev. Fluid Mech. 26, 23 (1994).

${ }^{17}$ Z.-S. She, E. Jackson, and S. A. Orszag, "Structure and dynamics of homogeneous turbulence: models and simulations," Proc. R. Soc. London, Ser. A 434, 101 (1991). 